This document is the Accepted Manuscript version of a Published Work that appeared in final form in British Journal of Community Nursing, copyright @ MA Healthcare, after peer review and technical editing by the publisher. To access the final edited and published work see https://www.magonlinelibrary.com/doi/10.12968/bjcn.2018.23.Sup9.S6 


\title{
Understanding venous leg ulcers (VLS)
}

\begin{abstract}
Venous leg ulcers are open lesions between the knee and the ankle joint, which occur in the presence of venous insufficiency. There are theories to explain the causes of venous insufficiency which ultimately leads to venous hypertension and can result in leg ulceration. Although many patients present with evidence of venous hypertension, others do not, except for the manifestation of the ulcer. Although this is the case, there are risk factors associated with venous insufficiency and a holistic approach must be taken in order to influence the management approach of venous leg ulceration. This article discusses venous insufficiency as a disease process and explores the nursing assessment process, when assessing venous leg ulcers related to venous insufficiency.
\end{abstract}

Key words

Venous insufficiency, venous hypertension, venous ulceration

\section{Definition and epidemiology of venous leg ulcers:}

Venous leg ulcers are open lesions between the knee and the ankle joint (the gaiter area). They tend to occur on both lateral and medial aspect of the leg), which occur in the presence of venous insufficiency (Scottish Intercollegiate Guidelines Network [SIGN] 2010; Agale, 2013; Harding et al., 2015). They are also known as varicose or stasis ulcers, which are usually superficial, with poorly defined margins, a granulating wound bed and moderate to high exudate (Adeyi et al., 2009; Harding et al, 2015). They are a common, recurring condition (SIGN, 2010), with an estimated prevalence of 730,000 in the UK (Guest et al., 2015). There are other types of leg ulcers; however, venous leg ulcers account for 40-85\% (SIGN, 2010; Agale, 2013; Sarkar \& Ballantyne, 2000). This prevalence increases with age (Agale, 2013), rising to 20 per 1000 in people over the age of 80 (Royal College of Nursing [RCN], 2000). They occur in people from all socioeconomic backgrounds, however; evidence indicates that they take longer to heal, with a higher recurrence rate in people from lower socioeconomic backgrounds (SIGN, 2010), with a recurring risk between 26 and 69\% in 12 months (Nelson \& Bell-Syer, 2014).

Venous insufficiency is a result of venous hypertension. This is caused by valvular incompetence in deep veins, causing superficial veins to distend and stretch to accommodate the additional blood flow, as the valves are unable to close, leading to retrograde blood flow (Grey et al., 2006; Agale, 2013; Comerota \& Lurie, 2015). Despite reports on structural and biochemical abnormalities on vein walls, the primary cause of valvular incompetence remains unknown (Meissner et al., 2007). However, approximately $10 \%$ of the European and North American population have a valvular incompetence, with $0.2 \%$ developing venous ulceration (Grey et al., 2006).

Although valvular incompetence is considered as the major cause of venous hypertension, dysfunctional in the calf muscle pump also precipitates venous hypertension (Grey et al., 2006; O'Brien et al., 2012). The calf muscle pump is dependent on a full range of motion of the ankle joint and foot pump. Therefore, restricted movement of the ankle joint reduces calf muscle contraction leading to persistent hydrostatic venous pressure, which results in venous hypertension (Comerota 
\& Lurie, 2015; Harding et al., 2015). Most patients with venous leg ulcers are noted to have a significant reduction in ankle range of motion (Comerota \& Lurie, 2015; O’Brien et al., 2012).

Deep veins:

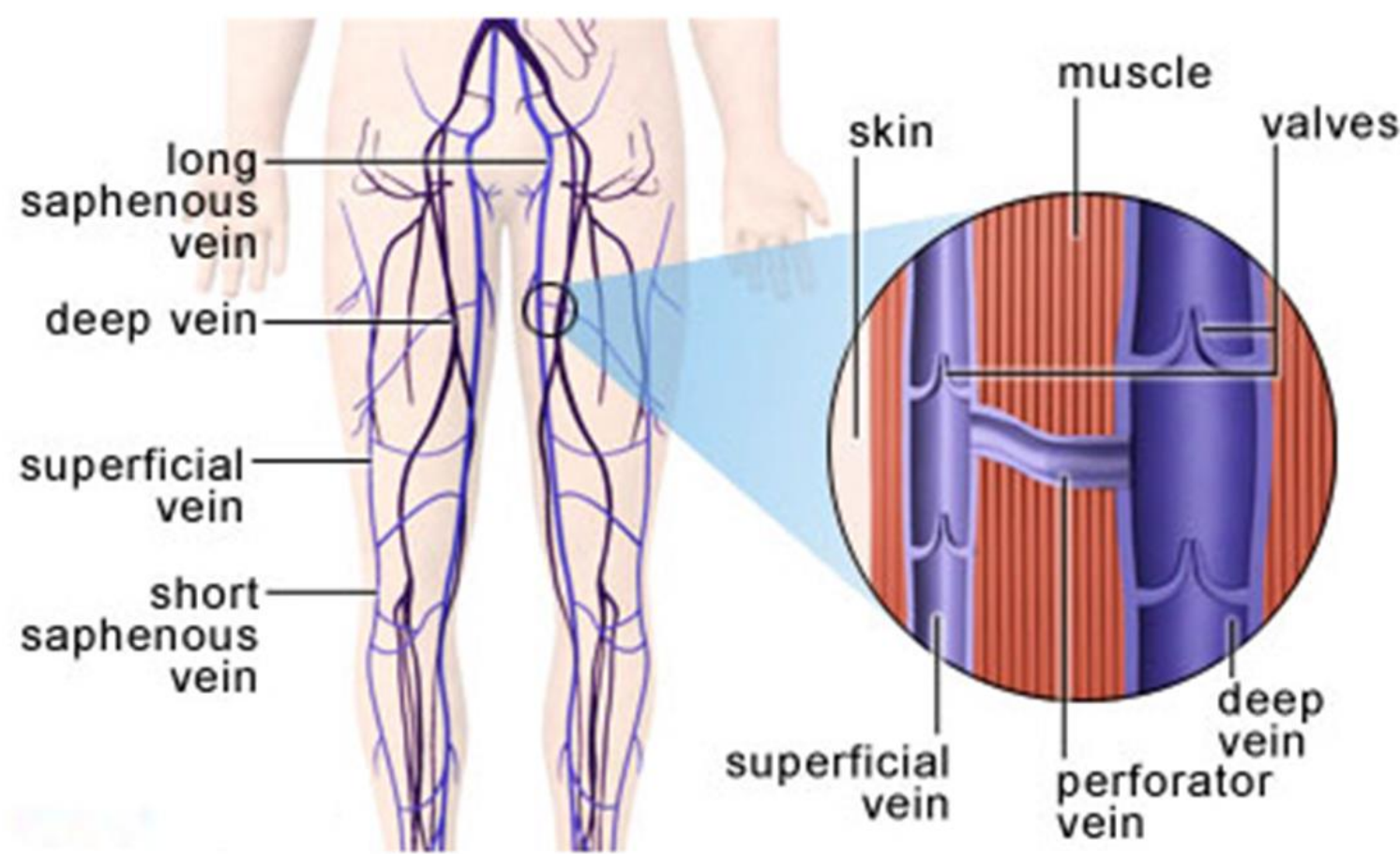

Calf muscle pump:

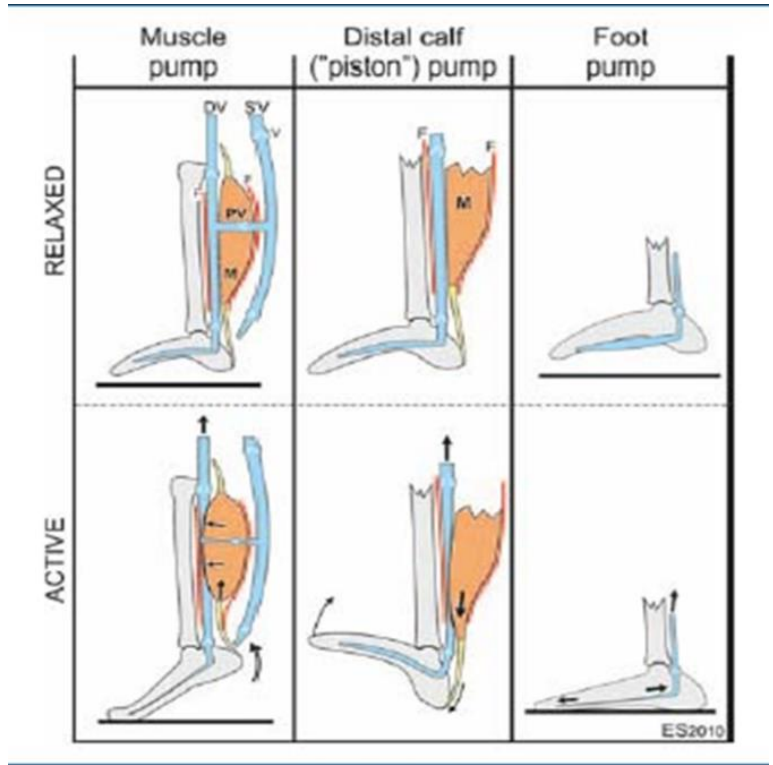

\section{Theories underpinning venous leg ulcers:}

Despite venous hypertension being linked to either valvular incompetence or calf muscle pump deficiency, there are theories which aim to explain the cause of venous ulceration (Liu et al., 2011; Gonsalves, 2003). These theories have been challenged by many studies, as venous ulceration is regarded as a complex cascade of events, with a number of cellular and humeral mechanisms linking 
it to venous hypertension (Meissner et al, 2007; Comerota \& Lurie, 2015). As a result, a unified theory of the pathophysiology remains speculative with a lack of understanding of venous ulceration aetiology (Siddiqui et al., 1995).

\section{Fibrin cuff theory}

The fibrin cuff theory argues that due to increased venous pressure, fibrinogen (a factor essential for clot formation) leaks from the capillaries (Goldstein \& Weingarten, 2001). This forms a peripcapillary fibrin cuff which inhibits the diffusion of oxygen and nutrients, leading to tissue damage and impaired wound healing (Vasudevan, 2014). However, this theory has been largely discounted as fibrin was considered insignificant as a barrier to diffusion as previously thought, despite being an evident cause of venous hypertension (Liu et al., 2011).

\section{White cell entrapment theory}

As a result of fibrin cuffs, white blood cells are trapped in capillary endothelium (Liu et al., 2001; Gonsalves, 2003). The decrease in venous flow in capillaries leads to an accumulation of white cells, which releases proteolytic enzymes (enzymes responsible for degrading necrotic tissue following cell breakdown) as well as interfere with oxygenation that causes endothelial damage (Gonsalves, 2003). Although the white cell entrapment theory is an acknowledged part of venous ulcer formation in people with venous hypertension, it is only a part of a complex cascade (Comerota \& Lurie, 2015; Meissner et al., 2007).

\section{Growth factor 'trap' theory}

Venous hypertension has been noted to cause macromolecules (such as protein and carbohydrates) to leak into the dermis and trap growth factors (Falanga \& Eaglstein, 1993). Growth factors are then unable to repair damaged tissue, or may be degraded by proteolytic enzymes, thus resulting in venous ulceration due to poor tissue regeneration (Vasudevan, 2014). Fibrin cuff has also been noted of presenting a barrier to the movement of growth factors necessary for tissue integrity and repair, resulting in ulcer formation (Falanga \& Eaglstein, 1993; Gonsalves, 2003).

\section{Signs of venous hypertension:}

Despite venous ulceration being the physical attribute of venous insufficiency and the above mentioned theories, it is also worth noting venous ulceration is a manifestation of venous hypertension, which is responsible for most venous pathology of the leg including those listed below (Liu et al., 2011; Meissner, 2007; Beebe-Dimmer, 2004).

\section{Varícose veîns}

Due to reversed blood flow in the lower limbs, some of the subcutaneous veins become dilated, once they are more than 4 millimiters, they are referred to as varicose veins (Beebe-Dimmer et al., 2004; National Institute for Health and Care Excellence [NICE], 2013). In some patients, venous hypertension does not present with varicose veins, instead it presents with hyphenwebs, which are defined as intradermal varices measuring between 1 millimetre and 3millimeters in diameter (Beebe-Dimmer et al, 2004).

Varicose veins: 


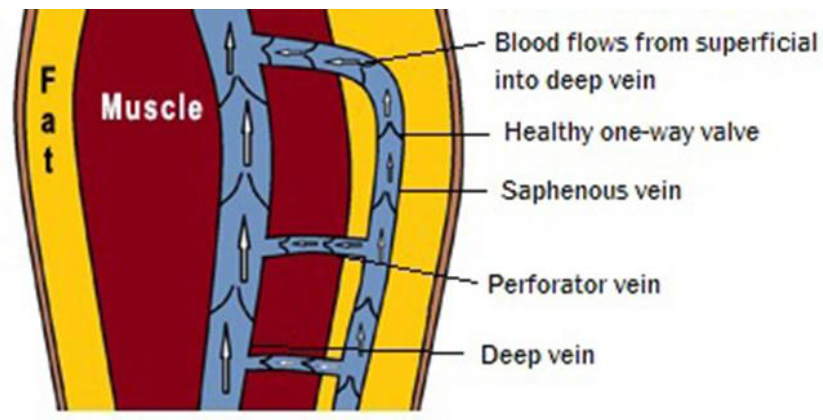

Blood Flow in Healthy Saphenous and Perforator Veins

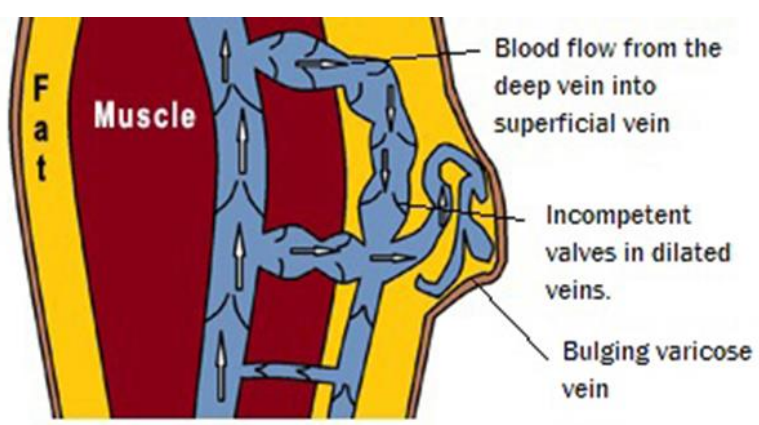

Blood Flow in Dilated

Sphenous and Perforator Veins

\section{Haemosiderin staining}

Following on from capillary distention and widening of endothelial pores due to venous hypertension, red blood cells escape with the blood plasma, and die within the interstitial space. This causes a reddish brown discolouration of the skin as they release haemoglobin (Bennett, 1998; Grey et al., 2006; Comerota \& Lurie, 2015).

\section{Ankle flare}

Venous hypertension may also present with ankle flare (also known as corona phlebectatica). This is a fan-shaped pattern of dilated veins around the malleolus, medial (inside) or lateral (outside) aspect of the foot or ankle (Antignani et al., 2012; Wright \& Fitridge, 2013; Harding et al., 2015).

\section{Atrophie blanche}

As a result of venous insufficiency, capillaries can be occluded in the middle and deep dermis causing atrophie blanche in a healed ulcer (Afsaneh et al., 2014; Ngan \& Elghblawi, 2017), which is mainly characterised by ivory-white depressed atrophic plaques, prominent red dots due to enlarged capillaries and hyperpigmentation due to haemosiderin staining (Ngan \& Elghblawi, 2017).

\section{Venous oedema}

Venous hypertension leads to leaking of fluid from the capillaries into the interstitial space, as a result of increased capillary permeability (Topham \& Mortimer, 2002). This fluid will present as a swelling of the limb which indents if finger pressure is applied, terming the condition pitting oedema (Hofman, 1998). Pitting oedema in some patients is not present in the morning; however, it may present or be worse towards the of the day as a result of limb dependence (Grey et al., 2006). Limb, or more specifically leg dependency, refers to the legs dangling, which will reduce venous return and increase capillary hydrostatic pressure, pushing fluid into the tissues. Hence, this type of oedema is known as dependence oedema. Hettrick (2009) offers a useful review of lower extremity gravitydependent oedema.

\section{Venous eczema}

Venous eczema, is a type of eczema related to venous reflux and inflamed varicose veins due to venous hypertension (Bradbury \& Pappas, 2006). It is usually characterised by itching, erythematous, blistering, weeping or scaling skin eruption, which is distinct from cellulitis (Grey et al., 2006; Harding et al., 2015; Wingfield, 2012). Venous eczema is also referred to as stasis dermatitis or gravitational eczema (Bradbury \& Pappas, 2006). 


\section{Lipodemasclerosis}

Lipodemasclerosis are changes to the lower limbs in patients with venous insufficiency, which are characterised by inflammation of the subcutaneous tissue, with two-thirds of the affected population being obese (Duffill, 2008). The subcutaneous tissue will be replaced by fibrosis which will present as woody induration (hardening), which may lead the leg to assuming the shape of an inverted champagne bottle or chicken drum stick (wide at the knee and very narrow at the ankle) (Bennett, 1999; Herouy et al., 1998; Grey et al., 2006; Harding et al., 2015). Lipodemasclerotic areas are prone to ulceration as a result of minor trauma due to loss of papillary structures in the dermoepidermal junction zone (area between the epidermis and the dermis) due to tissue fibrosis (Herouy et al., 1998; Wright \& Fitridge, 2013).

\section{Who is at risk of venous insufficiency and venous leg ulcers?}

Venous insufficiency, which precipitates venous hypertension can be influenced by a number of factors which are considered to be risk factors (Fowkes et al., 2001; Scott et al., 1995; Grey et al., 2006; Criqui et al., 2007; Comerota \& Lurie, 2015) such as:

\section{Deep vein thrombosis (DVT)}

Patients with a history of deep vein thrombosis are at an increased risk of developing venous insufficiency, as a result of damaged valves which will lead to backflow of blood in the veins at the point of damage (Agale, 2013; Wounds UK, 2016).

\section{Obesity}

Evidence suggests that being obese increases the amount of pressure exerted to veins in the legs (Scott et al., 1995; Beebe-Dimmer et al., 2005; Comerota \& Lurie, 2015). However, Fowkes et al., (2001) argues that, it is not known whether being obese merely accentuates the development of venous disease in those who are already susceptible to the condition. Yet, Agale (2013) agues, obesity leads to reduced mobility which results in inadequate functioning of the calf muscle pump; thus resulting in venous insufficiency.

\section{Pregnancy}

Pregnancy has been argued to be a risk factor of developing venous disease, with a positive relationship between prevalence and increase in number of pregnancies (Fowkes et al., 2001). Two theories have been suggested to support this argument: 1 . an increase venous pressure as a result of iliac vein compression in later stages of pregnancy 2 . a decrease in venous wall smooth muscle tone in response to hormonal changes especially estrogen (Staffa, 2002; Beebe-Dimmer et al., 2005). Despite, the two theories above some studies noted by Fowekes et al, (2001) have argued that there is no correlation between pregnancy and developing venous disease.

\section{Family history}

Multiple studies and reports have emphasised the correlation between family history of venous insufficiency and the development of venous disease (Criqui et al., 2007; Chi \& Raffetto, 2015; Staffa, 2002). From a study evaluated by Staffa (2002), hereditary venous disease incidence account for approximately $50 \%$ of all cases. 


\section{Extended periods of standing}

Evaluated studies by Fowkes et al., (2001), Staffa (2002) and Beebe-Dimmer et al., (2005) indicate the risk of developing venous disease in individuals occupying employment which requires them to spend long periods standing, as this impact on venous return leading to venous hypertension over time.

\section{Gender}

The female gender has been reported to be closely linked to venous disease (Fowkes et al., 2001; Agale, 2013), with a male to female prevalence ratio of 1.5 to 3.5 respectively (Staffa, 2002).

Age

Staffa (2002) notes, a list of epidemiological studies which confirm the relationship between age and an increased risk of developing venous disease of the lower leg, with a greater than $50 \%$ prevalence in individuals above the age of 70 , this could be influenced by reduced mobility and natural occurrence of venous disease (Adeyi et al., 2009). In addition to the above Criqui et al, (2007) also identified that there were no significant ethnic differences between these relationships; however, the disease was noted to be less severe in African-American woman.

\section{Trauma or surgery to the lower limbs}

A history of venous and leg trauma, such as fractured bones may cause DVT or impair the calf muscle pump (Wounds UK, 2016). There is lack of evidence supporting prolonged venous patency after venous reconstruction following trauma (Zamir et al., 1998). In addition, a study cited by Criqui et al, (2007) noted serious lower limb trauma to be a significant risk factor for venous disease.

\section{Calf muscle pump failure}

Calf muscle pump failure can be a result of paralysis, immobility, sleeping in a chair with leg dependant for prolonged periods of time and fixed ankle joints (Agale, 2015), adversely this impacts on venous return leading to increased venous pressure.

\section{Assessment of venous leg ulcers (VLU)}

In the presence of underlying venous disease, minor injury or trauma such as skin tear can result in VLU (Wounds UK, 2016). In an unfortunate event of a VLU developing, the patient needs to be assessed holistically. As numerous factors can lead to lower leg ulceration, in some cases it is essential that a multidisciplinary approach is adopted (Agale, 2012) in developing the care plan and predicting expected outcomes (Harding et al, 2015). This will enable an accurate diagnosis, avoid inappropriate treatments which may result in wound deterioration, delay wound healing or even harm the patient (Posnett \& Franks, 2008; Agale, 2012). The accurate diagnosis is enabled by a clinical assessment which includes past medical history, physical examination of the patient, leg and skin, investigations and modalities of treatments (Adeyi et al., 2009). Evidence has shown, many VLUs taking longer to heal, due to a lack of diagnosis and poor treatment choices (Posnett \& Franks, 2008; Guest et al., 2015).

\section{Clinical history}

Patient assessment must address ulcer history and medical history of previous treatments of venous disease, as the management of VLUs is often influenced by multiple comorbidities (SIGN, 2010; Dogra \& Sarangal, 2014). Clinical history taking will assist with the identification of any underlying 
venous disease and any other associated diseases, which may influence decision making and ultimately impact the outcome of the VLU management plan (RCN, 2000). For example, malnutrition, poor personal hygiene, intravenous drug abuse, and coexisting medical conditions such as diabetes mellitus, peripheral vascular disease, rheumatoid arthritis and vasculitis (SIGN, 2010; Dogra \& Sarangal, 2014).

\section{Physical examination}

As part of physical examination, the patient's general health status must be established by assessing baseline blood pressure, heart rate, blood glucose level and body mass index (RCN, 2006). It must also include, lower limb skin assessment, wound assessment and vascular assessment (Lindsay \& White, 2008; Chamanga, 2016).

\section{Skin and lower limb assessment}

Irrespective of the leg presenting with the ulcer, both legs must be assessed as part of differential diagnosis. The differential diagnosis will be examining for the presence of pitting or non-pitting oedema, signs of venous hypertension which will include: varicose veins, hemosiderin pigmentation, varicose eczema, atrophie blanche, and lipodermatosclerosis (RCN, 2006; SIGN; 2010; Dogra \& Sarangal, 2014).

\section{Wound assessment}

Wound assessment, must include site, size, depth, edge, margins, base, presentation of the surrounding skin (hyperkeratosis), clinical signs of infection (heat, inflammation, pain, increased exudate and malodour), wound edges (flat, punched out, rolled, circular or jagged edges) (RCN, 2006; SIGN, 2010; Lindsay \& white, 2008).

\section{Vascular assessment}

Following the skin and wound assessment, it is essential to exclude compromised arterial blood flow by doing an ankle brachial pressure index (ABPI) (RCN, 2006; SIGN; 2010). The assessment will indicate if the patient can be treated with the standard venous ulcer treatment (Compression therapy). The clinically acceptable ABPI readings are between 0.8-1.3 (Harding et al, 2015). Doppler sounds can also be used to determine the level of arterial function, with a traditional handheld Doppler machine presenting with three sounds: triphasic, biphasic and monophasic (Donnelly et al., 2000). The more advanced machines produce an electrogadiogram (ECG) waveform with accompanying notes advising whether arterial functioning is either normal or abnormal (Chamanga, 2016).

\section{Challenges commonly associated with VLUS}

Despite all the VLU management processes mentioned above, patients with VLUs are at high risk of developing cellulitis as a result of venous oedema in the interstitial space, creating an environment conducive for bacterial infection to grow, as the open wound provides a portal of entry (Swartz, 2004; Wingfield, 2009). Cellulitis is an acute bacterial infection of the dermis and subcutaneous tissue, which usually affects one leg and rarely bilateral (Swartz, 2004). It may appear as a swollen, red area of the skin that feels hot and tender to touch and which may spread rapidly (Baxter \& McGregor, 2001; Phoenix et al., 2012). Traditionally, compression therapy was contraindicated in patients with the fear of pushing the infection from interstitial space into blood circulation and the associated pain (Baxter \& McGregor, 2001; Clinical Resource Efficiency Support Team [CREST], 2005; Wingfield, 2012). However, a panel of experts argued that as long as the patients' pain levels allow, 
compression therapy must be continued as it helps prevent further lymphatic damage (WoundsUK, 2016).

Venous eczema is also known to be a risk factor for the opportunistic development of lower limb cellulitis (Wingfield, 2009). Eczema in VLU management is usually caused by two factors: Endogenous or Exogenous - independently or in conjunction with each other (Lo, 2010; Cameron, 1998; Lawton, 2009). Endogenous factors are internal factors, such as proteolytic enzymes caused by venous hypertension, whilst exogenous factors being reactions to external stimuli, such as irritation and allergic or sensitivity contact (Cameron, 1998). Evidence suggests that venous eczema can be managed by the application of topical steroids or potassium permanganate solution (Cameron, 1998; Wingfield, 2012; Wounds UK, 2016). All steroids are prescribed as either ointments or creams. Ointments are suitable for dry eczema, whilst creams are suitable for wet eczema - the latter absorbing quickly before being "washed away". There is a multitude of advice on application of topical steroids which appears to be subjective and varies from one clinical area to the other (Lagos \& Maibach, 1998; Lawton, 2009; NICE, 2004). Therefore, the application of topical steroids in VLU management must be guided by local guidelines, the discretion of health professional and their individual patient (NICE, 2004).

\section{Conclusion}

Venous insufficiency leads to venous hypertension which in some cases manifests as VLUs among other clinical presentation. There are risk factors associated with the development of venous hypertension which are age, weight and family history to mention a few. The prevalence of VLUs increases with the aging process and it has been noted to be high in Europe and North America. In the unfortunate event of a patient developing a venous leg ulcer, it requires holistic assessment and in some cases multidisciplinary interventions. It is worth noting that patient with venous insufficiency and VLUs are at risk of developing cellulitis and venous eczema. Both these complications will need to be treated according to each individual practitioner clinical area's guidelines.

\section{References}

Afsaneh, A. et al. 9 other writers, (2014) Atrophie Blanchie: Is it Associated with Venous Disease or Livedoid Vasculopathy? Advances in Skin \& Wound Care, 27(11): 518-524.

Anderson, I. \& Smith, G. (2014) Compression Made Easy. WoundsUK, 10(3)1-6.

Ashby, R. I., Gabe, R., Ali, S., et al., (2014) Clinical and cost-effectiveness of compression hosiery versus compression bandages in treatment of venous leg ulcers (VenUS IV): a randomised controlled trial. Lancet, 383(9920): 871-879.

Antignani, P. L., Carpentier, P. H., Cornu-Thenard, A., Flour, M., Partsch, H., Bare, E. \& UHL, J. F. (2012) UIP consensus on corona Phlebeltatica. International Angiology, 13 (3) 217-218.

Adeyi, A. et al. (2009) 'Leg Ulcers In Older People: A Review Of Management Leg Ulcers In Older People: A Review Of Management', British Journal of Medical Practitioners, 2(3): 21-28. 
Agale, S.V. (2013) 'Chronic leg ulcers: epidemiology, aetiopathogenesis, and management', Ulcers, 2013.

Beebe-dimmer, J. L. Pfeirer, J. R., Engle, J. S. \& Schottenfeld, D. (2004) The epidemiology of chronic venous insufficiency and varicose veins. Annual Epidemiology, 15:175184.

Baxter, H. \& McGregor, F. (2001) Understanding and managing cellulitis. Nursing Standard, 15(44): 50-56.

Bradbury, A. W. \& Pappas, P. J. (2006) Chronic venous insufficiency, varicose veins, lymphedema, and arteriovenous fistulas. In Davies \& Brophy, Vascular Surgery. Springer, London.

Bennett, A. (1999) Leg ulcer assessment and diagnosis. Nursing \& Residential Care, 1 (6): 346-352.

Cameron, J. (1998) Skin care for patients with chronic leg ulcers. Journal of Wound Care, 7(8):459462.

Chamanga, E. T. (2016) Managing leg ulcers in Primary care. Nursing in Practice, 68-71.

Chi, Y. and Raffetto, J.D. (2015) 'Venous leg ulceration pathophysiology and evidence based treatment', Vascular Medicine, 20(2): 168-181.

Comerota, A. and Lurie, F. (2015) 'Pathogenesis of venous ulcer', Seminars in vascular surgery. Elsevier.

CREST (2005) Guidelines on the management of Cellulitis in Adults [online] http://www.acutemed.co.uk/docs/Cellulitis\%20guidelines,\%20CREST,\%2005.pdf

Criqui, M. H., Denenberg, J. O., Bergan, J., Langer, R. D. \& Fronek, A. (2007) Risk Factors for Chronic Venous Disease: the San Diego Population Study. Journal of Vascular Surgery, 46(2): 331-337.

Cheng, M., Changa, D. W. \& Patel, K. M. (2016) Principles and practice of lymphoedema surgery. Elsevier: London.

Dogra, S. \& Sarangal, R. (2014) Summary of recommendations for leg ulcers. Indian Dermatology Online Journal; 5(3): 400-407.

Donnelly. R, Hinwood. D, \& London. N. J. M. (2000) Non-invasive methods of arterial and venous assessment. British Medical Journal; 320:696-701.

Davies, J., Bull, R., Farrelly, I., Wakelin, M. (2008) Improving the calf pump using home-based exercises for patients with chronic venous disease. WoundsUK, 4(3):48-58.

Duffill, M. (2008) Lipodermatosclerosis. DermNet NZ https://www.dermnetnz.org/topics/lipodermatosclerosis

Falanga, V. \& Eaglstein, W. H. (1993) The trap hypothesis of venous ulceration. Lancet, 341:10061008. 
Fletcher, J. Moffatt, C., Partsch, H., Vowden, K. \& Vowden, P. (2013) Principles of compression in venous disease: a practitioner's guide to treatment and prevention of venous leg ulcers. Wounds International.

Felty, C. L. \& Rooke, T. W. (2005) Compression Therapy for chronic venous insufficiency. Seminars in Vascular Surgery, 18: 36-40.

Fowkes, F. G. R., Evans, C. J. \& Lee, A. J. (2001) Prevalence and Risk Factors of Chronic Venous Insufficiency. Angiology, 52(1) S5-S15.

Franks, P., Barker, J., Collier, M. et al (7 others). (2016) Management of patients with venous leg ulcer: challenges and current best practice, Journal of Wound Care, 25; 6, Suppl, 1-67.

Grey, J.E., Enoch, S. and Harding, K.G. (2006) ABC of Wound Healing-Venous and Arterial Leg Ulcers, BMJ, 332(7537): 347-350.

Gonsalves, G. F. (2003) Venous leg ulcers. Techniques in vascular and interventional radiology. 6(3):132-136.

Goldstein, E.J. C. \& Weingarten, M. S. (2001) State of the art treatment of chronic venous disease. Clinical Infectious Diseases, 23(6)949-954.

GUEST, J.F., AYOUB, N., MCILWRAITH, T., UCHEGBU, I., GERRISH, A., WEIDLICH, D., VOWDEN, K. and VOWDEN, P., 2015. Health economic burden that wounds impose on the National Health Service in the UK. BMJ open, 5(12), pp. e009283-2015-009283

Guest, J. F., Ayoub, N., Mcilwrait, T., Uchegbu, I., Gerrish, A., Weidlich, D., Vowden, K. \& Vowden, P., (2015). Health economic burden that wounds impose on the National Health Service in th UK. BMJ open, $5(2): 12$.

Hofman, D. (1998) Oedema and the management of venous ulcers. Journal of Wound Care, 7(7): 345

Harding, K. et al. (2015) 'Simplifying venous leg ulcer management: consensus recommendations'. Wounds International.

Herouy, Y. et al (8 others) (1998) Lipodermatosclerosis is characterised by elevated expression and activation of matrix metalloproteinases: Implications for venous ulcer formation. Journal of Investigative Dermatology, 111: 822-827.

Hettrick H (2009) The science of compression therapy for chronic venous insufficiency edema. The Journal of the American College of Certified Wound Specialists 1,1, 20-24

10.1016/i.jcws.2008.10.002 (last access 25 Match 2018)

Iglesias, C.P., Nelson, E.A., Cullum, N.A. et al. (2004) VenUS I: a randomised controlled trial of two types of bandage for treating venous leg ulcers. Health Technology Assessment, 8(29): 1-105.

Johnson, S. (2002) Compression hosiery in the prevention and treatment of venous leg ulcers. Journal of Tissue Viability, 12(2):67-74. 
Lindsay E, White R (2008) (eds). Leg ulcers and problems of the lower limb: A Holistic Approach. WoundsUK.

Lagos, B. R. \& Maibach, H. I. (1998) Frequency of application of topical corticosteroids an overview. British Journal of Dermatology, 139: 763-766.

Lawton, S. (2009) Assessing and treating adult patients with eczema. Nursing Standard, 23(43): 4956.

Liu, Y.C., Margolis, D.J. \& Isseroff, R.R. (2011) 'Does inflammation have a role in the pathogenesis of venous ulcers?: a critical review of the evidence', Journal of Investigative Dermatology, 131(4) 818827.

Lo, K. (2010) Practical approach for “Eczema”. The Hong Kong Medical Diary, 15 (11):5-7.

Miteva, M., Romanelli, P. \& Kersner, R. S. (2010) Lipodermatosclerosis. Dermatologic Therapy, 23: 375-388.

Mosti, G. \& Partsch, H. (2018) A new two component system turning an elastic bandage into an inelastic compression device: Interface pressure, stiffness, and haemodynamic effectiveness. European Journal of Endovascular Surgery, 55: 126-131.

Mosti, G. (2018) Venous ulcer treatment requires inelastic compression. Phlebologie, 47;7-12.

Meissner, M.H. et al. (2007) 'The hemodynamics and diagnosis of venous disease', Journal of Vascular Surgery, 46(6) S4-S24.

Nair, B. (2014) Compression therapy for venous leg ulcers. Indian Dermatology Online Journal, 5(3): 378-382.

Nelson, E. A., Mari, R. \& Vowden, K. (2008) Intermittent pneumatic compression for treating venous leg ulcers. Cochrane Database Systematic Review, 16(2): CD001899

Nelson, E.A. and Bell-Syer, S.E. (2014) 'Compression for preventing recurrence of venous ulcers', The Cochrane Library, .

Nelson, E. A., Hillman, A. \& Thomas, K. (2014) Intermittent pneumatic compression for treating venous leg ulcers. Cochrane Database Systematic Review, 12(5): CD001899

NICE, (2015) Leg ulcer-venous. Available at: http://cks.nice.org.uk/leg-ulcer-venous

NICE, (2004) Frequency of application of topical corticosteroids for atopic eczema. [Online] https://www.nice.org.uk/guidance/ta81/resources/frequency-of-application-of-topicalcorticosteroids-for-atopic-eczema-2294813945797/

NICE, (2013) Varicose veins: diagnosis and management. https://www.nice.org.uk/guidance/cg168 Ngan, V. \& Elghblawi, E. (2017) Atrophie Blanchie. DermNet NZ https://www.dermnetnz.org/topics/atrophie-blanche/ 
O'Meara, S., Cullum, N., Nelson, E.A., \& Dumville, J.C. (2012) Compression for venous leg ulcers. Cochrane Database of Systematic Reviews. 11:1-191.

O'Brien, J.A. (2010) A Pilot Study Assessing the Feasibility of a Home-Based Progressive Resistance Exercise Program and Trend Toward Healing Rates for Patients with Venous Leg Ulcers. Available at: http://eprints.qut.edu.au/43615/

O'Brien, J. A., Edwards, H. E., Finlayson, K. J. \& Kerr, G. (2012) Understanding the relationship between the calf muscle pump, ankle range of motion and healing for adults with venous leg ulcers: a review of literature. Wound Practice and Research, 20(2): 80-85.

Phoenix, G., Das, S. \& Joshi, M. (2012) Diagnosis and management of cellulitis. BMJ clinical reviews, 345(4955):1-8.

Posnett, J. \& Franks, P. J. (2008) The burden of chronic wounds in the UK. Nursing Times; 104: 44-45.

Royal College of Nursing. (2000) The management of patients with venous leg ulcers. Audit Protocol. Royal College of Nursing.

Royal College of Nursing. (2006) Clinical practice guidelines: The management of patients with venous leg ulcers. RCN, London.

Regmi, S. \& Regmi, K. (2012) 'Best practice in the management of venous leg ulcers', Nursing Standard, 26(32),56, 58, 60.

Sarkar, P.K. \& Ballantyne, S. (2000) 'Management of leg ulcers', Postgraduate Medical Journal, 76(901),674-682.

Scottish Intercollegiate Guidelines Network (2010) Management of chronic venous leg ulcers: 120. www.sign.ac.uk/pdf/sign120.pdf

Siddiqui, A., Galiano, R. D. \& Mustoe, T. (1995) Theory of growth factors and ulcer healing. Vascular Medicine Review, 6: 79-86.

Staffa, R. (2002) Chronic venous insufficiency-epidemiology. Bratisl Lek Listy; 103(4-5): 166-168.

Scott, T. E., LaMorte, W. W., Gorin, G. R. \& Menzoian, J. O. (1995) Risk factors for chronic venous insufficiency: A dual case-control study. Journal of Vascular Surgery, 22(5)622-628.

Swartz, M. N. (2004) Cellulitis. New England Journal of Medicine. 350: 904-912.

Topham, E. J. \& Mortimer, P. S. (2002) Chronic lower limb oedema. Clinical Medicine, 2(1): 28-31.

Vasudevan, B. (2014) Venous leg ulcers: Pathophysiology and classification. Indian Journal of Dermatology, 5(3): 366-370.

Wingfield, C. (2009) Lower Limb Cellulitis: a dermatological perspective. Wounds UK, 5(2) 26-36.

Wingfield, C. (2012) Diagnosing and managing lower limb cellulitis. Nursing Times, 108(27), 18-21. 
Wounds UK. (2016) Best Practice Statement: Holistic management of venous leg ulceration. London: Wounds UK.

Wright, N. and Fitridge, R. (2013) Varicose veins - natural history, assessment and management. Australian Family Physician 42(6), 380-384.

Zamir, G., Berlartky, Y., Rivkind, A., Anner, H. \& Wolf, Y. G. (1998) Results of reconstruction in major pelvic and extremity venous injuries. Journal of Vascular Surgery, 28: 901-908. 